TRANSACTIONS OF THE

AMERICAN MATHEMATICAL SOCIETY

Volume 358, Number 10, October 2006, Pages 4553-4567

S 0002-9947(06)03860-8

Article electronically published on April 11, 2006

\title{
SPECTRA OF QUANTIZED HYPERALGEBRAS
}

\author{
WILLIAM CHIN AND LEONID KROP
}

\begin{abstract}
We describe the prime and primitive spectra for quantized enveloping algebras at roots of 1 in characteristic zero in terms of the prime spectrum of the underlying enveloping algebra. Our methods come from the theory of Hopf algebra crossed products. For primitive ideals we obtain an analogue of Duflo's Theorem, which says that every primitive ideal is the annihilator of a simple highest weight module. This depends on an extension of Lusztig's tensor product theorem.
\end{abstract}

\section{INTRODUCTION}

We describe the prime and primitive spectra of the restricted specialization of the quantized enveloping algebra $U_{\zeta}$, where $\zeta$ is a root of unity of odd order $\ell$ (prime to 3 in type $G_{2}$ ) associated to a semisimple complex Lie algebra $\mathfrak{g}$ of rank $n$ as in e.g. [Lu1, Lu2, Lu4, Li, An], working over a field k of characteristic zero. We refer to $U_{\zeta}$ as the hyperalgebra because it is defined using a divided power form in a way which mimics the usual hyperalgebra construction for an affine algebraic group.

There is a Hopf algebra map Fr : $U_{\zeta} \rightarrow U$, where $U$ is the ordinary enveloping algebra, known as the quantum Frobenius map. It is known [Li, An that the Hopf kernel of $\mathrm{Fr}$ is the quantized restricted enveloping algebra $u_{\zeta}$, which is a normal sub-Hopf algebra of $U_{\zeta}$. Furthermore $U_{\zeta}$ is isomorphic as an algebra to the crossed product $u_{\zeta} \#_{t} U$ as k-algebras for some 2-cocycle $t: U \otimes U \rightarrow u_{\zeta}$. This is explicitly shown in [An], follows from the general results in [Sch], and was earlier pointed out informally by Takeuchi. See [Mo, Do for details about crossed products. The crossed product decomposition is an important tool for our study of prime and primitive ideals.

The crossed product structure allows us to describe the prime spectrum of $U_{\zeta}$ in terms of the prime spectra of $u_{\zeta}$ and $U$. The structure of the latter as a topological space is only partially understood [So, though it is explicitly described in rank one $\mathrm{NG}$ and in type $A_{2} \mathrm{Ca}$. We think of the crossed product structure as being a ring-theoretic explanation of the Lusztig twisted tensor product theorem [Lu1, Li]. Indeed, we use it to give a different proof of the tensor product theorem which moreover applies to all simple modules. This is then applied to prove a version of Duflo's Theorem, which states that every primitive ideal is the annihilator of a simple highest weight module. This result invites the study of primitive spectra for $U_{\zeta}$ in connection with Weyl group orbit geometry, as has been carried

Received by the editors November 5, 2003 and, in revised form, September 27, 2004.

2000 Mathematics Subject Classification. Primary 16W35, 16W30, 17B37, 81R50.

This work was supported in part by a grant from the University Research Council of DePaul University. 
out extensively for $U$; see [Ja] (and [Jo] 8.5 .4 for more references). We note that the generic quantized version of Duflo's Theorem was proved by Joseph and Letzter; see [Jo].

In the second section we discuss $U_{\zeta}$ and its images modulo annihilators of a simple $u_{\zeta}$-module. The crossed product in the image is shown to be a tensor product with trivial action and cocycle, facts which depend on the Skolem-Noether Theorem and the vanishing of the Sweedler two-cocycle. We use this fact to show that the prime spectrum of $U_{\zeta}$ is homeomorphic to $\operatorname{Spec}(U) \times \operatorname{Spec}\left(u_{\zeta}\right)$ in section 3. We turn to primitive ideals in section 4 , first developing the theory of weights and simple highest weight modules. Here we allow for possibly infinite-dimensional simple modules, which are labeled by algebra maps from the Cartan part $U_{\zeta}^{0}$ to the base field $\mathrm{k}$. The resulting group of weights has a very different structure from the generic case. In passing, we give an expression for the primitive elements in $U_{\zeta}$, which is not used in what follows, but may be of independent interest since they are (in a Hopf-algebraic sense) a natural choice as generators. In section 4.2, assuming $\mathrm{k}$ algebraically closed, we prove an extension of Lusztig's tensor product theorem [Lu1, $\mathrm{CP}$, Li] to all simple modules for $U_{\zeta}$. We provide the version of Duflo's Theorem in the last subsection, 4.3.

The authors thank I. M. Musson for his suggestions and Z. Lin for useful discussions.

1.1. Notation and preliminaries. We adopt the notation and conventions for the quantized enveloping algebra $U_{\zeta}$ of rank $n$ at the primitive root of unity $\zeta$ of order $\ell$ as in [Lu1, 2,4] (also [An, Li]), working over a field $\mathrm{k}$ of characteristic zero. $\ell$ is odd and not divisible by 3 if there is a component of type $G_{2}$. The algebra $U_{\zeta}$ has generators $E_{i}, F_{i}, E_{i}^{(\ell)}, F_{i}^{(\ell)}, K_{i},\left(\begin{array}{c}K_{i} \\ \ell\end{array}\right), i=1, \ldots, n$. Let $\left(a_{i j}\right)$ be the symmetrizable Cartan matrix of finite type with integers $d_{i} \in\{1,2,3\}$ such that $d_{i} a_{i j}=d_{j} a_{j i}$ for all $i, j$. Let $\zeta_{i}=\zeta^{d_{i}}$. We invoke the relations $K_{i}^{\ell}=1$ (as done in e.g. Li]), and thus only consider type 1 modules. We shall use $\Delta$ and $\varepsilon$ for comultiplication and counit, using subscription to indicate the Hopf algebra in question.

Let $L(m)$ denote the finite-dimensional simple highest weight $U_{\zeta}$-module with restricted integral weight $m \in[0, \ell-1]^{n}$. Then the restriction of this module to $u_{\zeta}$ is simple, and every simple $u_{\zeta}$-module is of this form. The proof of these facts can be found in Lu1, Lu2] (cf. [CP, 11.2.10]) in the simply-laced case, and it is well known that they hold in general.

Working over $\mathbb{Z}\left[q, q^{-1}\right]$, let $q_{i}=q^{d_{i}}$. For $c, m \in \mathbb{Z}$ and $r, t \in \mathbb{Z}^{+}$set

$$
\begin{aligned}
{\left[\begin{array}{c}
m \\
t
\end{array}\right]_{q_{i}} } & =\prod_{s=1}^{t} \frac{q_{i}^{m-s+1}-q_{i}^{-(m-s+1)}}{q_{i}^{s}-q_{i}^{-s}}, \\
{\left[\begin{array}{c}
K_{i} ; c \\
t
\end{array}\right]_{q_{i}} } & =\prod_{s=1}^{t} \frac{K_{i} q_{i}^{c-s+1}-K_{i}^{-1} q_{i}^{-(c-s+1)}}{q_{i}^{s}-q_{i}^{-s}} .
\end{aligned}
$$

Specializing $q$ to $\zeta$, we have the corresponding elements $\left[\begin{array}{c}m \\ t\end{array}\right]_{\zeta_{i}} \in \mathrm{k}$ and $\left[\begin{array}{c}K_{i} ; c \\ t\end{array}\right]_{\zeta_{i}}$ $\in U_{\zeta}$. Thus $\left(\begin{array}{c}K_{i} \\ \ell\end{array}\right)=\left[\begin{array}{c}K_{i} ; 0 \\ \ell\end{array}\right]_{\zeta_{i}} \in U_{\zeta}$. 
We shall need several formulas for later use that we summarize here. Fix $1 \leq$ $i, j \leq n$. Then we have the defining conjugation relations

$$
\begin{aligned}
K_{i} E_{j} K_{i}^{-1} & =\zeta_{i}^{a_{i j}} E_{j}, \\
K_{i} F_{j} K_{i}^{-1} & =\zeta_{i}^{-a_{i j}} F_{j} .
\end{aligned}
$$

These relations imply

$$
\begin{aligned}
& \left(\begin{array}{c}
K_{i} \\
\ell
\end{array}\right) F_{j}^{(t)}=F_{j}^{(t)}\left(\begin{array}{c}
K_{i} ;-t a_{i j} \\
\ell
\end{array}\right), \\
& \left(\begin{array}{c}
K_{i} \\
\ell
\end{array}\right) E_{j}^{(t)}=E_{j}^{(t)}\left(\begin{array}{c}
K_{i} ; t a_{i j} \\
\ell
\end{array}\right) .
\end{aligned}
$$

The next equation is a simple verification:

$$
\left[\begin{array}{c}
m \\
t
\end{array}\right]_{q_{i}}=(-1)^{t}\left[\begin{array}{c}
-m+t-1 \\
t
\end{array}\right]_{q_{i}} .
$$

For $m \in \mathbb{Z}$, we write $m=m_{0}+m_{1} \ell \in \mathbb{Z}$ with unique $m_{0}, m_{1} \in \mathbb{Z}$ with $0 \leq m_{0} \leq$ $\ell-1$ and refer to this as the "short $\ell$-adic decomposition of $m$ ". We mention the well-known fact that

$$
\left[\begin{array}{c}
m \\
\ell
\end{array}\right]_{\zeta_{i}}=m_{1}
$$

see [Lu1, also [CP, 9.3.6].

Let $m$ be an integer with $0 \leq m \leq \ell-1$, and let $c=c_{0}+c_{1} \ell$ be the short $\ell$-adic decomposition of $c$. Then we have

$$
\begin{aligned}
& {\left[\begin{array}{c}
m-c \\
\ell
\end{array}\right]_{\zeta_{i}}=\left\{\begin{array}{l}
-c_{1} \text { if } m \geq c_{0}, \\
-\left(c_{1}+1\right) \text { if } m<c_{0},
\end{array}\right.} \\
& {\left[\begin{array}{c}
m+c \\
\ell
\end{array}\right]_{\zeta_{i}}=\left\{\begin{array}{l}
c_{1} \text { if } m+c_{0}<\ell, \\
c_{1}+1 \text { if } m+c_{0} \geq \ell
\end{array}\right.}
\end{aligned}
$$

The first equation, (1.5), of this last pair of equations can seen by applying equation (1.3) to obtain $\left[\begin{array}{c}m-c \\ \ell\end{array}\right]_{\zeta_{i}}=(-1)^{\ell}\left[\begin{array}{c}c_{1} \ell-\left(m-c_{0}\right)+\ell-1 \\ \ell\end{array}\right]_{\zeta_{i}}$. If $m-c_{0} \geq 0$, we get $(-1)^{\ell} c_{1}=-c_{1}$ using (1.4). If $m-c_{0}<0$, then $c_{1} \ell-\left(m-c_{0}\right)+\ell-1=\left(c_{1}+1\right) \ell+$ $c_{0}-m-1$ and we get $-\left(c_{1}+1\right)$ for the second case. The second equation, (1.6), is similar.

\section{Crossed Products}

We begin by briefly reviewing some results concerning cleft extensions and crossed products. Readers may wish to refer to [Mo, Do for more detailed discussions. Fix a subalgebra $A$ of a Hopf algebra $U$ and a right $U$-comodule algebra $T$. An extension of algebras $A \subset T$ is said to be cleft, or more precisely $U$-cleft, if there exists a convolution-invertible $U$-comodule map $\chi \in \operatorname{Hom}(U, T)$ and the subalgebra of coinvariants $T^{\mathrm{co} U}$ for $U$ equals $A$. Such a map $\chi$ is called a section. A result from DT is that $T$ is isomorphic to a crossed product $A \#{ }_{t} U$ for some action of $U$ on $A$ and invertible cocycle $t$ if and only if $T$ is $U$-cleft.

In [Do, it is shown that for a Hopf algebra $U$ and any $U$-cleft extension $T$ of the algebra $A$, any two sections $\chi^{\prime}, \chi \in \operatorname{Hom}(U, T)$ are related by $\chi^{\prime}=v * \chi$ for 
some convolution invertible $v \in \operatorname{Hom}(U, A)$. Recall also that corresponding to each section $\chi$, there is a k-isomorphism

$$
\tilde{\chi}: A \otimes U \rightarrow T
$$

given by $\tilde{\chi}(a \otimes y)=a \chi(y), a \in A, y \in U$, which is an algebra isomorphism when the tensor product is given the crossed product structure. The crossed product is denoted by $A \#_{t} U$ (with action and twisting $t$ depending on $\chi$ ) and the elements $a \otimes y$ are written $a \# y$ or $a \#_{t} y$. Using the second section, we affix ' and obtain an equivalent crossed product and isomorphism $\tilde{\chi}^{\prime}: A \#_{t^{\prime}} U \rightarrow T$ with new twisting $t^{\prime}$ and

$$
\tilde{\chi}^{\prime}\left(a \#_{t^{\prime}} y\right)=a \chi^{\prime}(y)=\sum a v\left(y_{1}\right) \chi\left(y_{2}\right)
$$

with $a \#_{t^{\prime}} y \in A \#_{t^{\prime}} U$. The corresponding algebra isomorphism $A \#_{t^{\prime}} U \rightarrow A \#_{t} U$ sends $a \#_{t^{\prime}} y$ to $\sum a v\left(y_{1}\right) \#_{t} y_{2}$ [Do, p. 3064].

We now look at $U_{\zeta}$ and fix notation. The Hopf algebra extension (see $\mathrm{An}$ )

$$
\mathrm{k} \rightarrow u_{\zeta} \rightarrow U_{\zeta} \stackrel{\mathrm{Fr}}{\rightarrow} U \rightarrow \mathrm{k}
$$

is cleft. Here $U=U(\mathfrak{g}), U_{\zeta}$ is a right $U$-comodule using ( $\left.\mathrm{id} \otimes \operatorname{Fr}\right) \Delta$ and $u_{\zeta}$ is the coinvariant subalgebra. The fact that $u_{\zeta}$ is the set of right coinvariants follows directly from the PBW theorem. We note for later use that $U_{\zeta}$ is also a left $U$ comodule using $(\operatorname{Fr} \otimes i d) \Delta$ and $u_{\zeta}$ is the left coinvariant subalgebra. An explicit section $\gamma: U \rightarrow U_{\zeta}$ is given by first specifying

$$
\gamma\left(e_{i}\right)=E_{i}^{(\ell)}, \gamma\left(f_{i}\right)=F_{i}^{(\ell)}, \gamma\left(h_{i}\right)=\left(\begin{array}{c}
K_{i} \\
\ell
\end{array}\right),
$$

where the $e$ 's, $f$ 's and $h$ 's are the usual generators for the enveloping algebra $\mathrm{An}$; we then extend to obtain three algebra maps $U^{+} \rightarrow U_{\zeta}^{+}, U^{-} \rightarrow U_{\zeta}^{-}$, and $U^{0} \rightarrow U_{\zeta}^{0}$. The definition of $\gamma$ is completed by tensoring, using the triangular decompositions

$$
\begin{aligned}
U & =U^{-} \otimes U^{0} \otimes U^{+}, \\
U_{\zeta} & =U_{\zeta}^{-} \otimes U_{\zeta}^{0} \otimes U_{\zeta}^{+},
\end{aligned}
$$

i.e., $\gamma\left(x^{-} x^{0} x^{+}\right)=\gamma\left(x^{-}\right) \gamma\left(x^{0}\right) \gamma\left(x^{+}\right)$for $x^{+} \in U^{+}, x^{0} \in U^{0}, x^{-} \in U^{-}$. It is very straightforward check that $\gamma$ is both a right and left $U$-comodule map. The section $\gamma$ corresponds to the crossed product $u_{\zeta} \#_{t} U \cong U_{\zeta}$ for some action of $U$ on $u_{\zeta}$ and some 2-cocycle $t: U \otimes U \rightarrow u_{\zeta}$.

We remark that it follows from results in $\underline{\mathrm{Sch}}$ that a section $U \rightarrow U_{\zeta}$ exists. Also, our section $\gamma$ differs slightly from the one in $\mathrm{An}$, since there the relations $K_{i}^{\ell}=1$ are not assumed.

Let $A$ be an algebra with crossed product action by a Hopf algebra $U$. Then $T=A \#_{t} U$ is a associative algebra. We briefly review some ring-theoretic facts and definitions. If $I$ is a $U$-invariant ideal of $A$, then it is standard and easy to check that $T J \subset J T=J \# T$ is an ideal of $T$. For an ideal $I$ of $A$, as usual (e.g. Ch), we put $(I: U)=\{a \in A \mid x . a \in I$, all $x \in U\}$, the largest $U$-invariant ideal of $A$ contained in $I$. A $U$-invariant ideal $J$ of $A$ is said to be $U$-prime if $K L \subset J$ implies $K \subset J$ or $L \subset J$, for all $U$-invariant ideals $L, K \subset A$.

The action of $U$ on $A$ is said to be inner (see [Mo or [Do]) if there exists a convolution-invertible $u \in \operatorname{Hom}_{\mathrm{k}}(U, A)$ such that y.a $=\sum u\left(y_{1}\right) a u^{-1}(y)$ for all $a \in A$ and $y \in U$. Here the action of $U$ is said to be implemented by $u$. Note that $u$ is not, in general, an algebra map. 
Lemma 1. Consider the crossed product $U_{\zeta}=u_{\zeta} \#{ }_{t} U$ as above. Then the $U$-prime ideals and the prime ideals of $u_{\zeta}$ coincide.

Proof. Let $\mathfrak{m}$ be a $U$-prime ideal of $u_{\zeta}$. Then by [Ch], $\mathfrak{m}$ is a prime ideal. Conversely, let $\mathfrak{m}$ be a prime ideal of $u_{\zeta}$. Then $(\mathfrak{m}: U)$ is a $U$-prime ideal. By [Ch] again, $(\mathfrak{m}: U)$ is actually a prime and hence maximal ideal. So $\mathfrak{m}=(\mathfrak{m}: U)$. This shows that every prime ideal of $u_{\zeta}$ is $U$-prime.

Proposition 1. Consider the crossed product $U_{\zeta}=u_{\zeta} \#_{t} U$ arising from the section $\gamma$ as above. Let $\mathfrak{m}$ be a prime ideal of $u_{\zeta}$ and set $R=u_{\zeta} / \mathfrak{m} . \quad$ Let $T=U_{\zeta} / \mathfrak{m} U_{\zeta}$ and let $\pi: U_{\zeta} \rightarrow T$ denote the natural map. Then

(a) $\chi=\pi \gamma \in \operatorname{Hom}(U, T)$ is a section which yields the crossed product

$$
T \cong R \#_{\bar{t}} U
$$

with cocycle $\bar{t}=\pi t: U \otimes U \rightarrow R$, via an isomorphism $\tilde{\chi}: R \#_{t} U \rightarrow T$ arising from the section $\chi$.

(b) There is another section $\chi^{\prime} \in H_{o m}(U, T)$ giving rise to the trivial crossed product

$$
T \cong R \otimes U
$$

via an isomorphism $\tilde{\chi}^{\prime}: R \otimes U \rightarrow T$ of cleft extensions of $R$.

(c) The corresponding isomorphism $\theta^{\prime}: R \#_{t} U \tilde{\rightarrow} R \otimes U$ is given by

$$
\theta^{\prime}(a \# y)=\sum a v\left(y_{(1)}\right) \otimes y_{(2)}
$$

for all $a \in R, y \in U$, for some $v \in \operatorname{Hom}_{\mathrm{k}}(U, R)$.

Proof. By the lemma $\mathfrak{m}$ is a $U$-invariant ideal. Therefore, as pointed out before Lemma $1, U_{\zeta} \mathfrak{m} \subseteq \mathfrak{m} U_{\zeta}=\mathfrak{m}{ }_{t} U$, so $\mathfrak{m} U_{\zeta}$ is an ideal of $U_{\zeta}$. Since $\mathfrak{m}$ is a trivial right $U$-comodule, $\mathfrak{m} U_{\zeta}$ is also a right $U$-subcomodule of $U_{\zeta}$. Hence $T$ inherits a $U$-comodule algebra structure.

(a): Plainly, $\chi$ is a section of $T$. Thus we have $T \cong R \#{ }_{t} U$ with cocycle $\bar{t}=\pi t$ : $U \otimes U \rightarrow R$.

(b) and (c): Obviously $R$ is a simple algebra; in fact $\mathfrak{m}$ is the annihilator of a finite-dimensional simple $u_{\zeta}$-module of dimension, say $n$. Since the simple modules are absolutely simple, $R$ is isomorphic to the algebra of $n \times n$ matrices over k.

The Skolem-Noether Theorem ([Sw, see also [Mo, 6.2]) asserts that the action of $U$ on $R$ is inner. By [Mo, 7.3] we can perform a change of basis to obtain $R \#_{t} U \cong R_{\tau}[U]$, a twisted product, with trivial action and a new cocycle $\tau$. The isomorphism is given explicitly by a map that is the identity on $R=R \# 1$ as follows:

$$
a \#_{t} y \longmapsto \sum a u^{-1}\left(y_{(1)}\right) \#_{\tau} y_{(2)}
$$

for all $y \in U, a \in R$, where the inner action is implemented by $u, u^{-1} \in \operatorname{Hom}(U, R)$ as in $[\mathrm{Mo}, 7.3]$.

Furthermore, the action of $U$ is now trivial, so $R$ is a (trivial) $U$-module. By [Mo, 7.1] or [Do, since $U$ is cocommutative, the cocycle $\tau$ has image in the center of $R$, namely $\mathrm{k}$. The reader may also check as an exercise that the twisted module condition quickly implies that, for a trivial action of any Hopf algebra, any cocycle has central values. Thus the Sweedler cocycle $\tau$ corresponds to a Lie cocycle ( $\underline{\mathrm{Sw}}$, 4.1 or 4.3]) with coefficients in the trivial module. Therefore $\tau$ is equivalent to a Sweedler coboundary by Whitehead's Second Lemma. Here we have used the equivalence of the Lie (and Hochschild) and Sweedler cohomologies in degree $2[\mathrm{Sw}$, 
section 4]. We conclude by [Do, 2.4] that $R_{\tau}[U]$ is isomorphic to a smash product with the same trivial action of $U$ on $R$; i.e, $R_{\tau}[U] \cong R \otimes U$. Thus $R \#_{t} U \cong R \otimes U$ by an isomorphism that is the identity on $R=R \# 1=R \otimes 1$.

The isomorphism $R \#{ }_{t} U \cong R \otimes U$ is given by

$$
a \# y \rightarrow \sum a v\left(y_{(1)}\right) \otimes y_{(2)},
$$

$a \in R, y \in U$, where $v=u * w \in \operatorname{Hom}(U, R)$ and $w$ is the map whose image is the coboundary $\tau$, by [Do, 2.4]. The corresponding section $\chi^{\prime}$ is given by $v * \chi$.

\section{Prime spectrum}

Theorem 1. $\operatorname{Spec}\left(U_{\zeta}\right)$ is homeomorphic to $\operatorname{Spec}(U) \times \operatorname{Spec}\left(u_{\zeta}\right)$.

Proof Fix a prime ideal $P \triangleleft U_{\zeta}$ and let $\mathfrak{m}=P \cap u_{\zeta}$, and put $R=u_{\zeta} / \mathfrak{m}$. Then $\mathfrak{m}$ is a $U$-prime, and hence maximal, ideal of $R$ by the lemma. As in $\mathrm{Ch}$, we define

$$
\operatorname{Spec}_{\mathfrak{m}}\left(U_{\zeta}\right)=\left\{Q \in \operatorname{Spec}\left(U_{\zeta}\right): Q \cap u_{\zeta}=\mathfrak{m}\right\} .
$$

Note that the sets $\operatorname{Spec}_{\mathfrak{m}}\left(U_{\zeta}\right)$ are the connected components of $\operatorname{Spec}\left(U_{\zeta}\right)$. By e.g. Ch] we have $\operatorname{Spec}_{\mathfrak{m}}\left(U_{\zeta}\right) \approx \operatorname{Spec}\left(\frac{U_{\zeta}}{\mathfrak{m} U_{\zeta}}\right) \approx \operatorname{Spec}\left(R \#_{t} U\right)$. By the proposition above we see that $\operatorname{Spec}_{\mathfrak{m}}\left(U_{\zeta}\right) \approx \operatorname{Spec}(R \# U) \approx \operatorname{Spec}(U)$. Thus we obtain a mapping $\pi: \operatorname{Spec}\left(U_{\zeta}\right) \rightarrow \operatorname{Spec}(U)$ that restricts to a homeomorphism on each $\operatorname{Spec}_{\mathfrak{m}}\left(U_{\zeta}\right)$.

To complete the proof, define the mapping $h: \operatorname{Spec}\left(U_{\zeta}\right) \rightarrow \operatorname{Spec}\left(u_{\zeta}\right) \times \operatorname{Spec}(U)$ by $h(P)=\left(P \cap u_{\zeta}\right) \times \pi(P)$. Clearly, $h$ maps $\operatorname{Spec}_{\mathfrak{m}}\left(U_{\zeta}\right)$ homeomorphically onto the connected component $\mathfrak{m} \times \operatorname{Spec}(U)$.

\section{Primitive spectrum}

\subsection{Simple highest weight modules.}

4.1.1. Weights. Let $U_{\zeta}^{0}$ denote the Hopf subalgebra of $U_{\zeta}$ generated by the $K_{i}$ and the $\left(\begin{array}{c}K_{i} \\ \ell\end{array}\right), i=1, \ldots, n$, which may also be constructed by specializing the corresponding subalgebra of the Lusztig form to $\zeta$. Also let $G$ denote the multiplicative group of group-likes generated by the $K_{i}$ 's. Since $U_{\zeta}^{0}$ is a pointed commutative and cocommutative Hopf algebra, by the Kostant structure theorem (cf. [Mo]) it is the tensor product of the (Hopf) algebras $\mathrm{k} G$ and the commutative enveloping algebra $U\left(P\left(U_{\zeta}^{0}\right)\right)$, with primitive generators $\left.P\left(U_{\zeta}^{0}\right)\right)$. On the other hand, restricting the mapping Fr to $U_{\zeta}^{0}$ and the section $\gamma$ to the subalgebra $U^{0}=\mathrm{k}\left[h_{1}, \ldots, h_{n}\right]$, we see that $U_{\zeta}^{0}$ is a crossed product $\mathrm{k} G \# \mathrm{k}\left[\left(\begin{array}{c}K_{1} \\ \ell\end{array}\right), \ldots,\left(\begin{array}{c}K_{n} \\ \ell\end{array}\right)\right]$ with trivial action and trivial cocycle. Thus

Proposition 2. $U_{\zeta}^{0}$ is the polynomial algebra generated over $\mathrm{k} G$ by the algebraically independent elements $\left(\begin{array}{c}K_{i} \\ \ell\end{array}\right), i=1, \ldots, n$.

The $\left(\begin{array}{c}K_{i} \\ \ell\end{array}\right)$ are not the primitive generators of $U_{\zeta}^{0}$; cf. Remark 1 following Proposition 4 below.

We consider weights to be algebra homomorphisms $\lambda: U_{\zeta}^{0} \rightarrow \mathrm{k}$. We let

$$
X=A g_{\mathrm{k}}\left(U_{\zeta}^{0}, \mathrm{k}\right)
$$


denote the set of weights. Weights $\lambda$ are specified by a pair of algebra maps $\lambda=$ $\left(\lambda_{0}, \lambda_{1}\right)$ with $\lambda_{0}: \mathrm{k} G \rightarrow \mathrm{k}$ and

$$
\lambda_{1}: \mathrm{k}\left[\left(\begin{array}{c}
K_{1} \\
\ell
\end{array}\right), \ldots,\left(\begin{array}{c}
K_{n} \\
\ell
\end{array}\right)\right] \rightarrow \mathrm{k}
$$

and thus are given by a pair of $n$-tuples

$$
\begin{aligned}
& \left(\lambda_{0,1}, \ldots, \lambda_{0, n}\right) \in\{0,1, \ldots, \ell-1\}^{n}, \\
& \left(\lambda_{1,1}, \ldots, \lambda_{1, n}\right) \in \mathrm{k}^{n},
\end{aligned}
$$

with $\lambda\left(K_{i}\right)=\zeta_{i}^{\lambda_{0 i}}$ and $\lambda\left(\left(\begin{array}{c}K_{i} \\ \ell\end{array}\right)\right)=\lambda_{1 i}$.

We say that $\lambda$ is a restricted weight if $\lambda_{1}=0$. By slight notational abuse we write $\lambda=\lambda_{0}$ in this case, and similarly $\lambda=\lambda_{1}$ if $\lambda_{0}=\varepsilon_{k G}$.

Integral weights. Let $m$ denote an $n$-tuple of integers $\left(m_{1}, \ldots, m_{n}\right) \in \mathbb{Z}^{n}$. We define an embedding ${ }^{\sim}: \mathbb{Z}^{n} \rightarrow X$ of sets by setting

$$
\begin{aligned}
\tilde{m}\left(K_{j}\right) & =\zeta_{j}^{m_{0 j}}, \\
\tilde{m}\left(\left(\begin{array}{c}
K_{j} \\
\ell
\end{array}\right)\right) & =m_{1 j},
\end{aligned}
$$

where $m_{0 j}$ and $m_{1 j}$ are the unique integers in the short $\ell$-adic decompositions $m_{0 j}+m_{1 j} \ell=m_{j}, j=1, \ldots, n$. In our above labeling of weights as pairs of $n$-tuples, this is equivalent to

$$
\begin{aligned}
& \tilde{m}_{0 j}=m_{0 j}, \\
& \tilde{m}_{1 j}=m_{1 j},
\end{aligned}
$$

$j=1, \ldots, n$.

We need to specify the convolution group structure on the set of weights which arises from the cocommutative Hopf algebra structure on $U_{\zeta}^{0}$. It is given by "carrying" from the finite abelian factor to the additive factor as we next state. We use * for the group operation on $X$.

Proposition 3. The group structure on $X=A \lg \left(U_{\zeta}^{0}, \mathrm{k}\right)$ is given by

$$
\left(\left(\lambda_{0}, \lambda_{1}\right) *\left(\mu_{0}, \mu_{1}\right)\right)_{j}=\left\{\begin{array}{l}
\left(\lambda_{0 j}+\mu_{0 j}, \lambda_{1 j}+\mu_{1 j}\right) \text { if } \lambda_{0 j}+\mu_{0 j}<\ell, \\
\left(\lambda_{0 j}+\mu_{0 j}-\ell, \lambda_{1 j}+\mu_{1 j}+1\right) \text { if } \lambda_{0 j}+\mu_{0 j} \geqslant \ell
\end{array}\right.
$$

for $\lambda, \mu \in X, j=1, \ldots, n$.

Proof. Let $\lambda, \mu \in X$. Since each $K_{i}$ is group-like,

$$
\begin{aligned}
\lambda * \mu\left(K_{i}\right) & =\lambda\left(K_{i}\right) \mu\left(K_{i}\right) \\
& =\zeta^{\lambda_{0 i}+\mu_{0 i}}=\left\{\begin{array}{l}
\zeta^{\lambda_{0 i}+\mu_{0 i}} \text { if } \lambda_{0 i}+\mu_{0 i}<\ell, \\
\zeta^{\lambda_{0 i}+\mu_{0 i}-\ell} \text { otherwise. }
\end{array}\right.
\end{aligned}
$$

Further, recall (e.g. [CP]) that $\Delta\left(\left(\begin{array}{c}K_{i} \\ \ell\end{array}\right)\right)=\sum_{j=0}^{\ell}\left(\begin{array}{c}K_{i} \\ \ell-j\end{array}\right) K_{i}^{-j} \otimes\left(\begin{array}{c}K_{i} \\ j\end{array}\right) K_{i}^{\ell-j}$, and observe that $\lambda\left(\left(\begin{array}{c}K_{i} \\ j\end{array}\right)\right)=\left(\begin{array}{c}\lambda_{0 i} \\ j\end{array}\right)_{\zeta_{i}}$ for all $j<\ell$. We infer that

$$
\lambda * \mu\left(\left(\begin{array}{c}
K_{i} \\
\ell
\end{array}\right)\right)=\lambda_{1 i}+w+\mu_{1 i}
$$


where the cross-term is $w=\sum_{j=1}^{\ell-1} \zeta_{i}^{-\left(\lambda_{0 i}+\mu_{0 i}\right) j}\left(\begin{array}{c}\lambda_{0 i} \\ \ell-j\end{array}\right)_{\zeta_{i}}\left(\begin{array}{c}\mu_{0 i} \\ j\end{array}\right)_{\zeta_{i}}$. The proof is completed by applying equation (4.4) below to deduce that $w=\left(\begin{array}{c}\lambda_{0 i}+\mu_{0 i} \\ \ell\end{array}\right)_{\zeta_{i}}$. By (1.4) we immediately obtain the desired result that $w=0$ or $w=1$ in cases as in the statement.

The following consequence is now straightforward. It explains the relationship between our weights and the integral weights as considered in [Lu1, Lu2], which were given as $n$-tuples of integers.

Corollary 1. The map ${ }^{\sim}: \mathbb{Z}^{n} \rightarrow X$ defined above is an embedding of abelian groups.

From now on we will use additive notation for the group operation on $X$. For future use we record the following easy facts.

Proposition 4. Let $\lambda=\left(\lambda_{0}, \lambda_{1}\right)$ and $\mu=\left(\mu_{0}, \mu_{1}\right)$ be weights and write $\lambda_{j}=$ $\left(\lambda_{0 j}, \lambda_{1 j}\right), j=1, \ldots, n$. The following equations hold:

$$
\begin{aligned}
(-\lambda)_{j} & =\left\{\begin{array}{l}
\left(\ell-\lambda_{0 j},-\lambda_{1 j}-1\right) \text { if } \lambda_{0 j}>0, \\
\left(0,-\lambda_{1 j}\right) \text { if } \lambda_{0 j}=0,
\end{array}\right. \\
(\lambda-\mu)_{j} & =\left\{\begin{array}{l}
\left(\ell+\lambda_{0 j}-\mu_{0 j}, \lambda_{1 j}-\mu_{1 j}-1\right) \text { if } \lambda_{0 j}<\mu_{0 j}, \\
\left(\lambda_{0 j}-\mu_{0 j}, \lambda_{1 j}-\mu_{1 j}\right) \text { if } \lambda_{0 j} \geq \mu_{0 j} .
\end{array}\right.
\end{aligned}
$$

Remark 1. We describe the primitive elements of $U_{\zeta}^{0}$. It suffices to work in rank 1 , so let $U=U_{\zeta}^{0}(\operatorname{sl}(2))$. Then up to a scalar multiple, $U$ has a unique primitive element

$$
\left(\begin{array}{c}
K \\
\ell
\end{array}\right)+\sum_{i=0}^{\ell-1} a_{i} K^{i}
$$

where

$$
a_{i}=\frac{1}{\ell^{2}} \sum_{j=0}^{\ell-1} j \zeta^{-i j}, i=0,1, \ldots, \ell-1 .
$$

Proof. The weight group $X=\operatorname{Alg}(U, k)$ can be written as the set of pairs $\left(\zeta^{i}, a\right)$, $a \in \mathrm{k}, i=0,1, \ldots, \ell-1$. Let $X^{\prime}$ be the group with the same underlying set but with group structure $\langle\zeta\rangle \times \alpha_{\mathrm{k}}$, where $\alpha_{\mathrm{k}}$ denotes the additive group of $\mathrm{k}$. Note that the coordinate Hopf algebra of $X^{\prime}$ is $U^{\prime}=\mathrm{k}[K, d]$, where $d$ is primitive and $K$ is a group-like of order $\ell$. Thus $\operatorname{Alg}\left(U^{\prime}, \mathrm{k}\right)$ is isomorphic to $X^{\prime}$, where the entries in the pairs correspond to evaluations at $K$ and $d$, respectively. It is easy to see that $X$ and $X^{\prime}$ are isomorphic via $\eta: X \rightarrow X^{\prime}$, where

$$
\eta\left(\left(\zeta^{i}, a\right)\right)=\left(\zeta^{i}, a+\frac{i}{\ell}\right) .
$$

Therefore we have an isomorphism of Hopf algebras $\eta^{*}: U^{\prime} \rightarrow U$. 
We proceed to calculate the primitive element $\eta^{*}(d)$. Let $\left(\zeta^{i}, a\right) \in X$. Evaluating, we have

$$
\begin{aligned}
& \left\langle\left(\zeta^{i}, a\right), \eta^{*}(d)\right\rangle \\
& =\left\langle\eta\left(\zeta^{i}, a\right), d\right\rangle \\
& =\left\langle\left(\zeta^{i}, a+\frac{i}{\ell}\right), d\right\rangle \\
& =a+\frac{i}{\ell} .
\end{aligned}
$$

On the other hand, since $\left(\begin{array}{c}K \\ \ell\end{array}\right)$ is primitive modulo the coradical $\mathrm{k}[K]$, we may write

$$
\eta^{*}(d)=b\left(\begin{array}{c}
K \\
\ell
\end{array}\right)+\sum_{i=0}^{\ell-1} a_{j} K^{j}
$$

for some $b, a_{j} \in \mathrm{k}$. Evaluating at $\left(\zeta^{i}, a\right)$ yields the system of $\ell$ equations

$$
b a+\sum_{j=0}^{\ell-1} a_{i} \zeta^{i j}=a+\frac{i}{\ell},
$$

$i=0, \ldots, \ell-1$. Setting $a=0,1$ immediately yields $b=1$. So we have the system of equations

$$
\sum_{j=0}^{\ell-1} a_{i} \zeta^{i j}=\frac{i}{\ell},
$$

which can be solved for the $a_{i}$ by inverting the Vandermonde matrix $\left(\zeta^{i j}\right)$. Its inverse is (fortunately, as pointed out to us by J. Angelos) $\frac{1}{\ell}\left(\zeta^{-i j}\right)$, as can easily be checked. This yields the asserted expressions for the coefficients $a_{i}$.

Since $U_{\zeta}^{0}$ has Krull dimension one and the coradical is finite, the k-space of primitives of $U_{\zeta}^{0}$ must be of dimension one. The uniqueness follows from the Kostant structure theorem; see [Mo].

4.1.2. Highest weight modules. We proceed to develop the theory of highest weight modules, following the standard strategy. The main difference is that we allow nonintegral weights, and the weights are an extension of the additive group of $\mathrm{k}^{n}$ by the finite abelian group $G=\left\langle K_{1}, \ldots, K_{n}\right\rangle$.

Definition 1. Let $V$ denote a $U_{\zeta}$-module. We say that $v \in V$ is a primitive vector of weight $\lambda \in X$ if

$$
\begin{gathered}
K_{i} . v=\lambda\left(K_{i}\right) v \\
\left(\begin{array}{c}
K_{i} \\
\ell
\end{array}\right) v=\lambda\left(\left(\begin{array}{c}
K_{i} \\
\ell
\end{array}\right)\right) v
\end{gathered}
$$

and $E_{i} v=E_{i}^{(\ell)} v=0$ for all $j$. We say that $V$ is a highest weight module if $V$ is generated by a primitive vector $v$.

In this case, $V$ is the sum of its weight spaces

$$
V_{\mu}=\left\{v \in V \mid x . v=\mu(x) v, \text { all } x \in U_{\zeta}^{0}\right\}
$$

for weights $\mu$.

We define a partial ordering on $X$ next. Let $\left(a_{i j}\right)$ be the symmetrizable Cartan matrix with entries $d_{i} a_{i j}=d_{j} a_{j i}$ in the standard notation. Simple roots are defined 
to be the restricted weights $\rho_{i}=\left(a_{1 i}, a_{2 i}, \ldots, a_{n i}\right)$, the $i^{\text {th }}$ column of $\left(a_{i j}\right), i=1, \ldots, n$. We say that $\lambda \preceq \mu$ if $\mu-\lambda$ is a nonegative integral linear combination of simple roots. This ordering is sensible because of the following proposition. Recall that we are using additive notation for the convolution group operation on $X$.

Proposition 5. Let $V$ be a $U_{\zeta}$-module and let $v \in V$ be a vector of weight $\lambda \in X$. Then $F_{i}^{(t)} v$ has weight $\lambda-t \rho_{i}$ and $E_{i}^{(t)} v$ has weight $\lambda+t \rho_{i}$ for all $t \in \mathbb{N}$.

Proof. Observe that

$$
\begin{aligned}
K_{j} F_{i}^{(t)} v & =\left(K_{j} F_{i}^{(t)} K_{j}^{-1}\right)\left(K_{j} v\right) \\
& =\left(\zeta_{j}^{-t a_{j i}} F_{i}^{(t)}\right)\left(\zeta_{j}^{\lambda_{0 j}} v\right) \\
& =\zeta_{j}^{\lambda_{0 j}-t a_{j i}} F_{i}^{(t)} v
\end{aligned}
$$

This establishes the first equation to be checked. We leave the analogous formula for $K_{j} E_{i}^{(t)} v$ to the reader.

The second pair of equations to be checked are

$$
\begin{aligned}
& \left(\begin{array}{c}
K_{j} \\
\ell
\end{array}\right) F_{i}^{(t)} v=\left(\lambda-t \rho_{i}\right) F_{i}^{(t)} v \\
& \left(\begin{array}{c}
K_{j} \\
\ell
\end{array}\right) E_{i}^{(t)} v=\left(\lambda+t \rho_{i}\right) E_{i}^{(t)} v .
\end{aligned}
$$

We begin by recalling a pair of identities (e.g. Lu2, g9-g10, p. 270]). Let $m, c \in \mathbb{N}$. Then

$$
\begin{aligned}
& {\left[\begin{array}{c}
K_{j} ;-c \\
\ell
\end{array}\right]_{q_{j}} }=\sum_{s=0}^{\ell}(-1)^{s} q_{j}^{c(\ell-s)}\left[\begin{array}{c}
c+s-1 \\
s
\end{array}\right]_{q_{j}} K_{j}^{s}\left[\begin{array}{c}
K_{j} \\
\ell-s
\end{array}\right]_{q_{j}}, \\
& {\left[\begin{array}{c}
K_{j} ; c \\
\ell
\end{array}\right]_{q_{j}}=\sum_{s=0}^{\ell} q_{j}^{c(\ell-s)}\left[\begin{array}{l}
c \\
s
\end{array}\right]_{q_{j}} K_{j}^{-s}\left[\begin{array}{c}
K_{j} \\
\ell-s
\end{array}\right]_{q_{j}} . }
\end{aligned}
$$

The next pair of formulas are obtained from these identities by specializing $K_{j}$ to $q_{j}^{m}:$

$$
\begin{aligned}
& {\left[\begin{array}{c}
m-c \\
\ell
\end{array}\right]_{q_{j}}=\sum_{s=0}^{\ell}(-1)^{s} q_{j}^{\ell c+s(m-c)}\left[\begin{array}{c}
c+s-1 \\
s
\end{array}\right]_{q_{j}}\left[\begin{array}{c}
m \\
\ell-s
\end{array}\right]_{q_{j}},} \\
& {\left[\begin{array}{c}
m+c \\
\ell
\end{array}\right]_{q_{j}}=\sum_{s=0}^{\ell} q_{j}^{\ell c-s(m+c)}\left[\begin{array}{c}
c \\
s
\end{array}\right]_{q_{j}}\left[\begin{array}{c}
m \\
\ell-s
\end{array}\right]_{q_{j}} .}
\end{aligned}
$$

If we put $m=\lambda_{0 j}$, the $s=0$ term in equations (4.3) and (4.4) is zero. Let $S$ denote the sum of the terms in equations (4.1) or (4.2) with $s>0$. Now using the obvious fact that $\left(\begin{array}{c}K_{j} \\ \ell-s\end{array}\right) v=\left[\begin{array}{c}\lambda_{0 j} \\ \ell-s\end{array}\right]_{\zeta_{j}} v$ for all $s>0$, we see that

$$
S . v=\left[\begin{array}{c}
\lambda_{0 j}-c \\
\ell
\end{array}\right]_{\zeta_{j}} v .
$$


Therefore

$$
\begin{aligned}
{\left[\begin{array}{c}
K_{j} ;-c \\
\ell
\end{array}\right]_{\zeta_{j}} v } & =\left(\begin{array}{c}
K_{j} \\
\ell
\end{array}\right) v+\left[\begin{array}{c}
\lambda_{0 j}-c \\
\ell
\end{array}\right]_{\zeta_{j}} v \\
& =\left(\lambda_{1 j}+\left[\begin{array}{c}
\lambda_{0 j}-c \\
\ell
\end{array}\right]_{\zeta_{j}}\right) v .
\end{aligned}
$$

Set $c=t a_{j i}$. Now by equation (1.1),

$$
\left(\begin{array}{c}
K_{j} \\
\ell
\end{array}\right) F^{(t)} \cdot v=\left(\lambda_{1 j}+\left[\begin{array}{c}
\lambda_{0 j}-c \\
\ell
\end{array}\right]_{\zeta_{j}}\right) F^{(t)} \cdot v .
$$

Writing the short $\ell$-adic decomposition $c=c_{0}+c_{1} \ell$, one has by 1.5

$$
\left[\begin{array}{c}
\lambda_{0 j}-c \\
\ell
\end{array}\right]_{\zeta_{j}}=\left\{\begin{array}{l}
-c_{1} \text { if } \lambda_{0 j} \geq c_{0}, \\
-\left(c_{1}+1\right) \text { otherwise }
\end{array}\right.
$$

This is the desired result for the action of the $F_{i}^{(t)}$. The analogous result for the action of $E_{i}^{(t)}$ is obtained similarly using equations (1.2), (1.6) and (4.4) in place of (1.1), (1.5) and (4.3).

Corollary 2. Let $v$ be a primitive vector of weight $\lambda \in X$. Then $\lambda$ is the highest weight in $U_{\zeta} v$ in the ordering $\preceq$ defined above.

4.1.3. Simple highest weight modules. Given a weight $\lambda$, we can construct a highest weight module for $U_{\zeta}$ by the usual Verma module construction. Using the standard argument, we find that there is a unique simple factor module which we denote by $L(\lambda)$ with primitive vector of weight $\lambda$, and every simple highest weight module is obtained in this way. Let

$$
T_{\lambda}=U_{\zeta} / \operatorname{ann}_{u_{\zeta}}(L(\lambda)) U_{\zeta}
$$

Clearly the representation $U_{\zeta} \rightarrow \operatorname{End}_{\mathrm{k}}(L(\lambda))$ factors through $T_{\lambda}$. We write

$$
\phi_{\lambda}: T_{\lambda} \rightarrow \operatorname{End}_{\mathrm{k}}(L(\lambda))
$$

for the representation of $T_{\lambda}$.

Consider a restricted weight $\lambda$; then $L(\lambda)$ is simple as a $u_{\zeta}$-module. Let

$$
R_{\lambda}=u_{\zeta} / \operatorname{ann}_{u_{\zeta}}(L(\lambda)) \cong \operatorname{End}_{\mathrm{k}}(L(\lambda)) .
$$

On the other hand, when $\lambda=\lambda_{1}$, then $\lambda$ factors through $U^{0}=\mathrm{k}\left[h_{1}, \ldots, h_{n}\right]$ and thus $\lambda$ corresponds in the obvious way to a weight $\bar{\lambda}$ for $U$. (This makes sense since $\lambda$ vanishes on $\operatorname{ker} \operatorname{Fr} \cap U_{\zeta}^{0}=\sum\left(K_{i}-1\right) U_{\zeta}^{0}$.) The corresponding simple $U$-module with highest weight $\bar{\lambda}=\bar{\lambda}_{1}$ shall be denoted by $L_{U}(\bar{\lambda})$, so that $L_{U}(\bar{\lambda})^{\mathrm{Fr}} \cong L(\lambda)$.

We will often drop the subscript $U$.

4.2. Tensor product theorem for simple modules. We recall the algebra map $\chi^{\prime}: U \rightarrow T$ from Proposition 1 .

Lemma 2. Let $\lambda$ be a restricted weight. Then $U$ acts trivially on $L(\lambda)$ via $\phi_{\lambda} \chi^{\prime}$ : $U \rightarrow \operatorname{End}_{\mathrm{k}}(L(\lambda))$, i.e., $\phi_{\lambda} \chi^{\prime}=\varepsilon_{U}$.

Proof. By Proposition 1, the elements of $\phi_{\lambda} \chi^{\prime}(U)$ commute with $R$ (as endomorphisms of $L(\lambda))$. Therefore $\phi_{\lambda} \chi^{\prime}$ sends $U$ to $\mathrm{k}=\operatorname{End}_{R}(L(\lambda)) \subset \operatorname{End}_{\mathrm{k}}(L(\lambda))$. 
Let $R$ and $U$ be rings, and let $L$ and $V$ be $R$ and $U$-modules, respectively. We give $L \otimes V$ (resp. $V \otimes L$ ) the structure of an $R \otimes U$-module (resp. $U \otimes R$ ), where the action is along tensor factors. If $L$ is a $U_{\zeta}$-module and $V$ is a $U$-module, we give $L \otimes V^{\mathrm{Fr}}$ and $V^{\mathrm{Fr}} \otimes L$ the usual module structure via the comultiplication of $U_{\zeta}$, with $V^{\mathrm{Fr}}$ considered as a $U_{\zeta}$-module along Fr. When $R=u_{\zeta} / \operatorname{ann}_{u_{\zeta}}(L)$ we have both module structures on the vector space $L \otimes V$, because $L$ can be viewed as a $U_{\zeta}$-module.

We shall assume $\mathrm{k}$ is algebraically closed for the remainder of the paper.

Theorem 2. (a) Every simple $U_{\zeta}$-module is isomorphic to $L \otimes V^{\mathrm{Fr}}$ for some simple highest weight module $L \cong L\left(\lambda_{0}\right)$ with restricted weight $\lambda_{0}$ and a simple $U$-module $V$ whose $U_{\zeta}$-module structure is via $\mathrm{Fr}$.

(b) Conversely, every module of this form, with $L$ and $V$ simple, is a simple $U_{\zeta^{-}}$ module.

(c) In addition, $V^{\mathrm{Fr}} \otimes L \cong L \otimes V^{\mathrm{Fr}} \cong L \otimes V \cong V \otimes L$.

Proof. Let $M$ be a simple $U_{\zeta}$-module. Then, as the annihilator $\mathfrak{m}=\operatorname{ann}_{u_{\zeta}}(M)$ is a prime ideal, $M$ is a semisimple $u_{\zeta}$-module with unique isotypic component, say $L=L\left(\lambda_{0}\right)$.

Let e be a primitive idempotent in $R=u_{\zeta} / \mathfrak{m}$. Then $L \cong R$ e and $\mathrm{e} R \mathrm{e}=\mathrm{k}$. As in Proposition 1 we have $T=U_{\zeta} / \mathfrak{m} U_{\zeta} \cong R \otimes U \cong M_{n}(U)$. Accordingly, eTe $\cong U$ and $T$ e is a projective indecomposable module for $T$. Clearly $L$ is a generator for $\bmod (R)$, so $T$ e is a generator for module category $\operatorname{Mod}(T)$.

By Morita equivalence, as left $T$-modules,

$$
\begin{aligned}
M & \cong T \mathrm{e} \otimes_{U} \mathrm{e} M \\
& \cong(L \otimes U) \otimes_{U} \mathrm{e} M \\
& \cong L \otimes \mathrm{e} M,
\end{aligned}
$$

where, in the last expression, $T=R \otimes U$ acts along tensor factors, and $\mathrm{e} M=V$ is the corresponding simple $\mathrm{e} T \mathrm{e} \cong U$-module.

Consider $L \otimes V$ as a $U_{\zeta}$-module via $\pi: U_{\zeta} \rightarrow T=R \otimes U$, and $L \otimes V^{\mathrm{Fr}}$ as a $U_{\zeta}$-module by comultiplying. Note that $L \otimes V^{\mathrm{Fr}}$ inherits a $T$-module structure since $\mathfrak{m} U_{\zeta}$ is a right $U$-subcomodule. We finish the proof of (a) by showing that $L \otimes V \cong L \otimes V^{\mathrm{Fr}}$. To do this it suffices to show that $\chi^{\prime}(U)$ only acts on the right factor $V^{\mathrm{Fr}}$, i.e.,

$$
\chi^{\prime}(a)\left(l \otimes v^{\mathrm{Fr}}\right)=l \otimes a v
$$

for all $a \in U, l \in L$ and $v \in V$. To show this, recall that $U_{\zeta}$ is a right $U$-comodule via $\rho_{\zeta}=(1 \otimes \mathrm{Fr}) \Delta_{U_{\zeta}}$. Thus $T$ inherits a right $U$-comodule with structure map denoted by $\rho: T \rightarrow T \otimes U$. We summarize these maps in the following diagram:

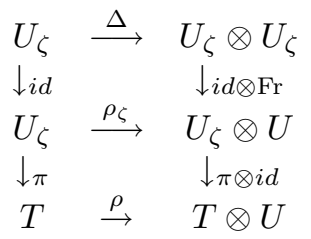

Similarly $T$ is a left $U$-comodule with structure map $\lambda: T \rightarrow U \otimes T$. 
We now compute

$$
\begin{aligned}
\chi^{\prime}(a)\left(l \otimes v^{\mathrm{Fr}}\right) & =\left(\rho \chi^{\prime}(a)\right)(l \otimes v) \\
& =\left(\chi^{\prime} \otimes i d_{T}\right) \Delta_{U}(a)(l \otimes v) \\
& =\left(\varepsilon_{U} \otimes i d_{T}\right) \Delta_{U}(a)(l \otimes v) \\
& =\left(i d_{U} \otimes a\right)(l \otimes v),
\end{aligned}
$$

where the second equality holds because $\chi^{\prime}$ is a right $U$-comodule map, and the third equality holds by the preceding lemma. This completes the proof of (a).

To prove (b), let $V$ be a simple $U$-module and $L$ a simple $u_{\zeta}$-module. By a well-known result $\mathrm{Qu}$ (since $\mathrm{k}$ is algebraically closed), $L$ and $V$ have trivial endomorphism rings $\mathrm{k}$. This fact, with a straightforward density theorem argument, shows that $L \otimes V$ is a simple $T$-module, with $R$ and $U$ acting along factors. Thus, as a $U_{\zeta}$-module via $\pi: U_{\zeta} \rightarrow T$, we see that $L \otimes V$ is a simple module.

To prove (c) we first claim that the right section $\chi^{\prime}$ is also a left $U$-comodule map. As in section 2, we have $U_{\zeta}=u_{\zeta} \gamma(U)$ and $u_{\zeta}$ is both left and right $U$-coinvariant for the respective left and right coactions $\lambda_{\zeta}=\left(\mathrm{Fr} \otimes i d_{U_{\zeta}}\right) \Delta_{U_{\zeta}}$ and $\rho_{\zeta}=\left(i d_{U_{\zeta}} \otimes \mathrm{Fr}\right) \Delta_{U_{\zeta}}$. Let $a \in u_{\zeta}$ and $y \in U$. Then in Sweedler notation,

$$
\rho_{\zeta}(a \gamma(y))=\sum a \gamma\left(y_{1}\right) \otimes y_{2},
$$

since $a$ is right coinvariant and $\gamma$ is a right $U$-comodule map. On the other hand,

$$
\lambda_{\zeta}(a \gamma(y))=\sum y_{1} \otimes a \gamma\left(y_{2}\right)
$$

since $a$ is left coinvariant and $\gamma$ is a right $U$-comodule map. Since $U$ is cocommutative, we see that $\lambda_{\zeta}=\tau \rho_{\zeta}$, where $\tau$ denotes the twist map. The same fact pushes down to $T$, i.e., $\lambda=\tau \rho$. Thus for any right comodule map $\varkappa: U \rightarrow T$,

$$
\begin{aligned}
\lambda \varkappa & =\tau \rho \varkappa \\
& =\tau\left(\varkappa \otimes i d_{U}\right) \rho \\
& =\left(i d_{U} \otimes \varkappa\right) \lambda .
\end{aligned}
$$

This establishes the claim that $\varkappa$ is a left $U$-comodule map.

By the left-comodule version of the argument just used in the last paragraph of the proof of part (a), we see that $V \otimes L \cong V^{\mathrm{Fr}} \otimes L$. Obviously, $V \otimes L \cong L \otimes V$ as $U_{\zeta}$-modules. Thus $V^{\mathrm{Fr}} \otimes L \cong V \otimes L \cong L \otimes V \cong L \otimes V^{\mathrm{Fr}}$. This completes the proof of (c) and the theorem.

Remark 2. The tensor symmetry in (c) follows from the quasi-tensor structure on $U_{\zeta}$ (see [Lu3]) when the modules are finite dimensional. We do not know this to be the case in general.

\subsection{An analog of Duflo's Theorem.}

Theorem 3. Every primitive ideal of $U_{\zeta}$ is the annihilator of a simple highest weight module.

Proof. Let $M$ be a simple left $U_{\zeta}$-module. Let $P$ denote the annihilator in $U_{\zeta}$ and set $m=u_{\zeta} \cap P$, which is a prime ideal of $u_{\zeta}$ by Lemma 1 . As a $U_{\zeta}$-module $M$ $\cong L \otimes V^{\mathrm{Fr}}$, where $L$ and $V$ are simple $u_{\zeta^{-}}$and $U$-modules, respectively. By Duflo's Theorem [Duf], $\operatorname{ann}_{U}(V)$ is the annihilator of a highest weight module for $U$, say $L_{U}\left(\bar{\lambda}_{1}\right)$. Therefore $\operatorname{ann}_{U_{\zeta}}\left(V^{\mathrm{Fr}}\right)=\operatorname{ann}_{U_{\zeta}}\left(L\left(\lambda_{1}\right)\right)$ for some weight $\lambda_{1}$ (notation as in section 4.1). 
Note also that $L$ is a simple highest weight module for $u_{\zeta}$, say of highest weight $\lambda_{0}$. Thus if $x \in L=L\left(\lambda_{0}\right)$ and $y \in L_{U}\left(\bar{\lambda}_{1}\right)$ are primitive vectors for the respective $u_{\zeta^{-}}$and $U$-module structures, then adopting the argument in [Lu1],

$$
\begin{aligned}
E_{i} \cdot(x \otimes y) & =\left(1 \otimes E_{i}+E_{i} \otimes K\right) \cdot(x \otimes y)=E_{i} \cdot x \otimes y=0, \\
E_{i}^{(\ell)} \cdot(x \otimes y) & =\left(1 \otimes E_{i}^{(\ell)}+E_{i}^{(\ell)} \otimes 1\right) \cdot(x \otimes y)=E_{i}^{(\ell)} \cdot x \otimes y+1+e_{i} \cdot y=0 \\
\text { and } & \\
K_{i} \cdot(x \otimes y) & =\lambda_{0}\left(K_{i}\right)(x \otimes y)=\lambda_{0, i}(x \otimes y), \\
\left(\begin{array}{c}
K_{i} \\
\ell
\end{array}\right) \cdot(x \otimes y) & =\lambda_{1}\left(\left(\begin{array}{c}
K_{i} \\
\ell
\end{array}\right)\right)(x \otimes y)=\lambda_{1, i}(x \otimes y)
\end{aligned}
$$

for all $i$. Hence $x \otimes y \in L \otimes L\left(\lambda_{1}\right)^{\mathrm{Fr}}$ is a primitive vector, and thus $L \otimes L_{U}\left(\bar{\lambda}_{1}\right)^{\mathrm{Fr}}$ is a simple highest weight module. Finally $\operatorname{ann}_{U_{\zeta}}(M)=\operatorname{ann}\left(L\left(\lambda_{0}\right) \otimes V^{\mathrm{Fr}}\right)$ can be computed using the Sweedler wedge in the coalgebra $U_{\zeta}$ (see [Mo] ) to be

$$
\begin{aligned}
& \operatorname{ann}_{U_{\zeta}}\left(L\left(\lambda_{0}\right)\right) \wedge \operatorname{ann}_{U_{\zeta}}\left(V^{\mathrm{Fr}}\right) \\
& =\operatorname{ann}_{U_{\zeta}}\left(L\left(\lambda_{0}\right)\right) \wedge \operatorname{ann}_{U_{\zeta}}\left(L_{U}\left(\bar{\lambda}_{1}\right)^{\mathrm{Fr}}\right) \\
& =\operatorname{ann}_{U_{\zeta}}\left(L\left(\lambda_{0}\right) \otimes L_{U}\left(\bar{\lambda}_{1}\right)^{\mathrm{Fr}}\right) .
\end{aligned}
$$

This completes the proof of the theorem.

\section{REFERENCES}

[An] N. Andruskiewitsch, Notes on Extensions of Hopf Algebras, Can. J. Math 48 (1996) 3-42. MR.1382474 (97c:16046)

[Ca] S. Catoiu, Prime ideals of the enveloping algebra $U\left(s l_{3}\right)$, Comm. Algebra 28 (2000), no. 2, 981-1027. MR1736777 (2001c:16048)

[Ch] W. Chin, Actions of solvable algebraic groups on noncommutative algebras, J. Contemp. Math. 124 (1992) 29-38. MR1144026 (93d:16032)

[CP] V. Chari and A. Pressley, A Guide to Quantum Groups, Camb. Univ. Press, Cambridge, 1994. MR 1300632 (95j:17010)

[Do] Y. Doi, Equivalent crossed products for a Hopf algebra, Comm. Alg. 17 (1989) 3053-3085. MR 1030610 (91k:16027)

[DT] Y. Doi and M Takeuchi, Cleft comodule algebras for a bialgebra, Comm. Alg. 14 (1986) 801-818. MR0834465 (87e:16025)

[Duf] M. Duflo, Sur la classification des idéaux primitifs dans l'algèbre enveloppante d'une algèbre de Lie semi-simple. Ann. Math. 105 (1977) 107-120. MR0430005 (55:3013)

[Ja] J. C. Jantzen, Einhüllende algebren halbeinfache Lie-Algebren, Springer, Berlin, 1983. MR0721170 (86c:17011)

[Jo] A. Joseph, Quantum Groups and Their Primitive Ideals, Springer, Berlin, 1995. MR 1315966 (96d:17015)

[Li] Z. Lin, Induced representations of Hopf algebras: applications to quantum groups at roots of 1, J. Alg. 154 (1993) 152-187. MR.1201918(94e:16046)

[Lu1] G. Lusztig, Modular representations and quantum groups, in "Classical Groups and Related Topics, pp. 55-77, Contemp. Math., Vol. 82, AMS, Providence, RI, 1989. MR0982278 (90a:16008)

[Lu2] G. Lusztig, Finite dimensional Hopf algebras arising from quantized universal enveloping algebras, J. AMS 3 (1990) 257-296. MR1013053 (91e:17009)

[Lu3] G. Lusztig, An Introduction to Quantum Groups, Prog. in Math. 110, Birkhäuser, Boston 1993. MR1227098 (94m:17016)

[Lu4] G. Lusztig, Quantum groups at roots of 1, Geom. Dedicata 35 (1990) 89-114. MR1066560 (91j:17018)

[Mo] S. Montgomery, Hopf Algebras and their Actions on Rings, CBMS Lecture Notes 82, AMS (1993). MR1243637 (94i:16019) 
[NG] Y. Nouazé, P. Gabriel, Idéaux premiers de l'algèbre enveloppante d'une algèbre de Lie nilpotente, J. Algebra 6 (1967) 77-99. MR0206064 (34:5889)

$[\mathrm{Qu}]$ D. Quillen, On the endomorphism ring of a simple module over an enveloping algebra, Proc. Amer. Math. Soc. vol. 21, issue 1 (1969), 171-172. MR0238892 (39:252)

[Sch] H.-J. Schneider, Normal bases and transitivity of crossed products for Hopf algebras, J. Alg. 152 (1992) 289-312. MR1194305 (93j:16032)

[Sw] M. Sweedler, Cohomology of algebras over Hopf algebras, Trans. A.M.S. 133 (1968) 205-239. MR.0224684 (37:283)

[So] W. Soergel, The prime spectrum of the enveloping algebra of a reductive Lie algebra, Math. Z., 204 (1990) 559-581. MR1062136 (91d:17015)

Department of Mathematics, DePaul University, Chicago, Illinois 60614

E-mail address: wchin@condor.depaul.edu

Department of Mathematics, DePaul University, Chicago, Illinois 60614

E-mail address: 1krop@condor.depaul.edu 